\title{
O uso da gastrostomia percutânea endoscópica
}

\author{
The use of percutaneous endoscopic gastrostomy
}

\author{
Marcos Ferreira MINICUCCI ${ }^{1}$ \\ Giovanni Faria SILVA ${ }^{1}$ \\ Mirna MATSUI ${ }^{1}$ \\ Roberto Minoru Tani INOUE \\ Leonardo Antônio Mamede ZORNOFF ${ }^{1}$ \\ Luiz Shiguero MATSUBARA ${ }^{1}$ \\ Sergio Alberto Rupp de PAIVA'
}

\section{R E S U M O}

A gastrostomia percutânea endoscópica foi introduzida na prática clínica como via alternativa de alimentação enteral. É procedimento que não necessita de anestesia ou laparotomia, como a gastrostomia cirúrgica. A gastrostomia percutânea endoscópica tem como objetivos a manutenção do aporte nutricional e a melhoria da qualidade de vida e sobrevida dos pacientes. É indicada quando o paciente necessita de dieta enteral por período superior a um mês. A freqüência de complicações varia de 1,0\% a 10,0\% e a mortalidade de 0,3\% a $1,0 \%$. É propósito deste trabalho abordar aspectos relevantes desse método de alimentação enteral, aprimorar seu manejo, bem como facilitar o manejo pelos pacientes que dele se utilizam. Para isso, vamos comentar as indicações e contra-indicações da gastrostomia percutânea endoscópica, técnica de colocação, material das sondas, antibiótico profilático, início de utilização, custo do procedimento, além de suas complicações, aspectos legais e éticos.

Termos de Indexação: acesso gastrointestinal, complicações, indicações e nutrição enteral, suporte nutricional.

\section{A B S T R A C T}

Percutaneous endoscopic gastrostomy is an alternative way to administer enteral diets. It is a procedure requiring no anesthetic or abdominal incision as required in open surgical gastrostomy. The main objective of percutaneous endoscopic gastrostomy is to prevent deterioration of the nutritional status and improve the quality of life and it is now the method of choice when the patient requires an enteral diet for more than a month. Complications occur in $1.0 \%$ to $10.0 \%$ of the cases and mortality in $0.3 \%$ to $1.0 \%$. This paper deals with aspects relevant to this method of enteral feeding, how to improve handling procedures and deal with the patients who use it, commenting on the indications and contraindications for its use, placement techniques, materials used for the probes, prophylactic antibiotics, start-up of its use and costs, in addition to discussing some of its complications and legal and ethical aspects.

Indexing terms: enteral access, complications, indications, enteral nutrition, nutritional support.

\footnotetext{
1 Departamento de Clínica Médica, Faculdade de Medicina de Botucatu, Universidade Estadual Paulista. Rubião Júnior, s/n, 18618-000, Botucatu, SP, Brasil. Correspondência para/Correspondence to: M.F. MINICUCCI.E-mail:<mminicucci@uol.com.br>.
} 


\section{N T R O D U Ç Ã O}

A gastrostomia percutânea endoscópica (GPE) foi introduzida como prática clínica em 1980, por Gauderer et al. ${ }^{1}$. Caracterizada como via alternativa de alimentação, utiliza a via enteral nos pacientes com trato gastrointestinal funcionante, mas com inabilidade ou impossibilidade de alimentação via oral. O número de GPE vem aumentando drasticamente nos últimos anos, tanto nos Estados Unidos quanto no Brasil.

É propósito deste trabalho apresentar e discutir as indicações, os objetivos e contra-indicações da GPE, técnica de colocação, material das sondas, antibiótico profilático, início de utilização, custo do procedimento, além de suas complicações, aspectos legais e éticos.

A gastrostomia é indicada quando o paciente necessita manter a via alternativa de alimentação enteral por mais de um mês ${ }^{2}$, pois a permanência da sonda nasoenteral, além desse período, aumenta o número de complicações ${ }^{3}$. A disfagia é o maior motivo da indicação da GPE. Essa dificuldade para deglutir pode ser devida a disfagias primárias: alterações peristálticas do esôfago que ocorrem nos acidentes vasculares encefálicos, doenças neurológicas, colagenoses e traumas; ou devida a disfagias secundárias: processos obstrutivos, como câncer de cabeça, pescoço e esôfago. Outras indicações mais raras encontradas na literatura são as correções de volvo gástrico, o manejo de medicações com paladar desagradável e a recirculação de bile drenada dos ductos biliares.

Skelly ${ }^{4}$, em artigo de revisão, descreve que $40,7 \%$ dos pacientes submetidos a GPE apresentavam acidente vascular encefálico, 34,7\% doenças neurovegetativas e 13,3\% câncer.

Um dos principais objetivos da GPE é a manutenção do aporte nutricional em pacientes com disfagia, nos quais a impossibilidade da ingestão via oral leva ao processo de desnutrição energético-protéica. Outros objetivos do procedimento são a hidratação, a prevenção de pneumonia aspirativa e o de proporcionar conforto e melhora da qualidade de vida e sobrevida dos pacientes. Assim, casos de hiperemese gravídica, anorexia nervosa e síndrome da imunodeficiência adquirida também podem beneficiar-se da GPE, lembrando-se de que os aspectos éticos devem ser avaliados nesses casos.

Em algumas situações, a GPE deve ser contra-indicada: obstrução do trato gastrointestinal ou dificuldade de aproximar a parede anterior do estômago à parede abdominal, como ocorre em ressecções gástricas prévias, ascite, hepatomegalia e obesidade, impedindo adequada transiluminação gástrica. As contra-indicações relativas englobam doenças neoplásicas, infiltrativas e inflamatórias do estômago e da parede abdominal, pela possibilidade de sua disseminação no trajeto da punção ${ }^{5}$, presença de cateteres de diálise peritoneal ${ }^{6}$, de derivação ventrículo-peritoneal pelo risco de peritonite ${ }^{7}$ e doenças respiratórias graves, pela dificuldade de realização da endoscopiå ${ }^{8}$.

Em relação à técnica de colocação da GPE, os componentes básicos do kit de GPE são: sonda de poliuretano ou silicone, cânula de punção, geralmente com válvula de segurança, bobina com fio duplo e placa de fixação externa. A fim de evitar complicações, deve-se fazer uma boa higiene oral e dentária, e utilizar técnicas cirúrgicas de assepsia. A GPE é executada com o paciente em posição de decúbito dorsal e requer a intervenção de um endoscopista e de um outro profissional para auxiliar no procedimento.

Após a inserção do endoscópio no estômago, é feita sua insuflação com ar, e procura-se localizar o melhor local para a punção, por meio de diafanoscopia. Segue-se com a infiltração de anestésico local em todas as camadas da parede abdominal e uma pequena incisão no local escolhido para a punção. A seguir, introduz-se a cânula de punção no estômago, sob o controle endoscópico, e, após retirar a agulha de punção, avança-se o fio duplo pela cânula até o estômago, onde é preso com uma pinça de biópsia (Figuras 1 e 2). Logo após, retira-se pela boca o fio preso à pinça, juntamente com o 
endoscópio. Externamente, passa-se o laço do fio duplo através do laço de fixação da sonda, atando-os entre si. Em seguida, puxando lentamente a extremidade do fio, coloca-se a sonda no interior do estômago. Puxa-se para fora, em conjunto, a cânula de punção e a sonda através das paredes gástrica e abdominal, até que o disco de retenção

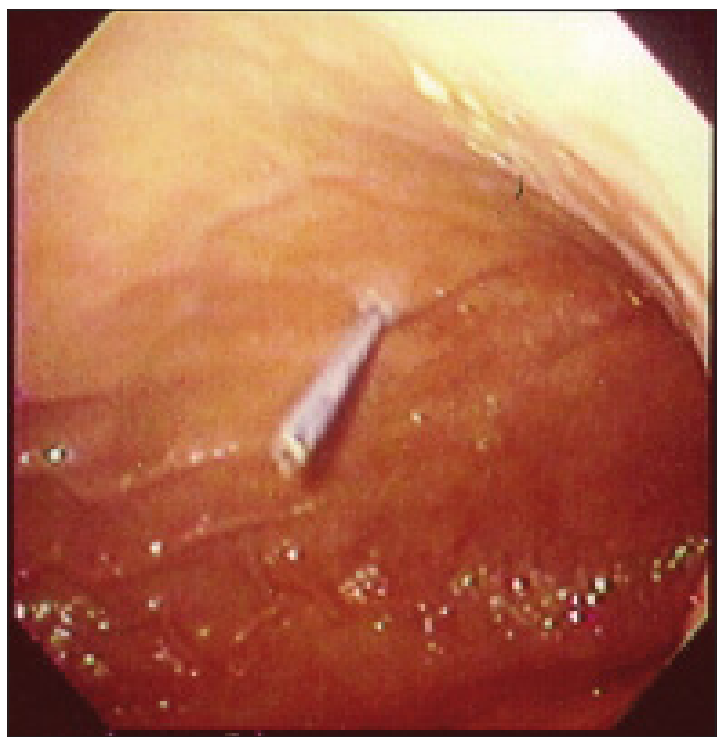

Figura 1. Aspecto endoscópico da parede do estômago; punção da parede anterior do estômago.
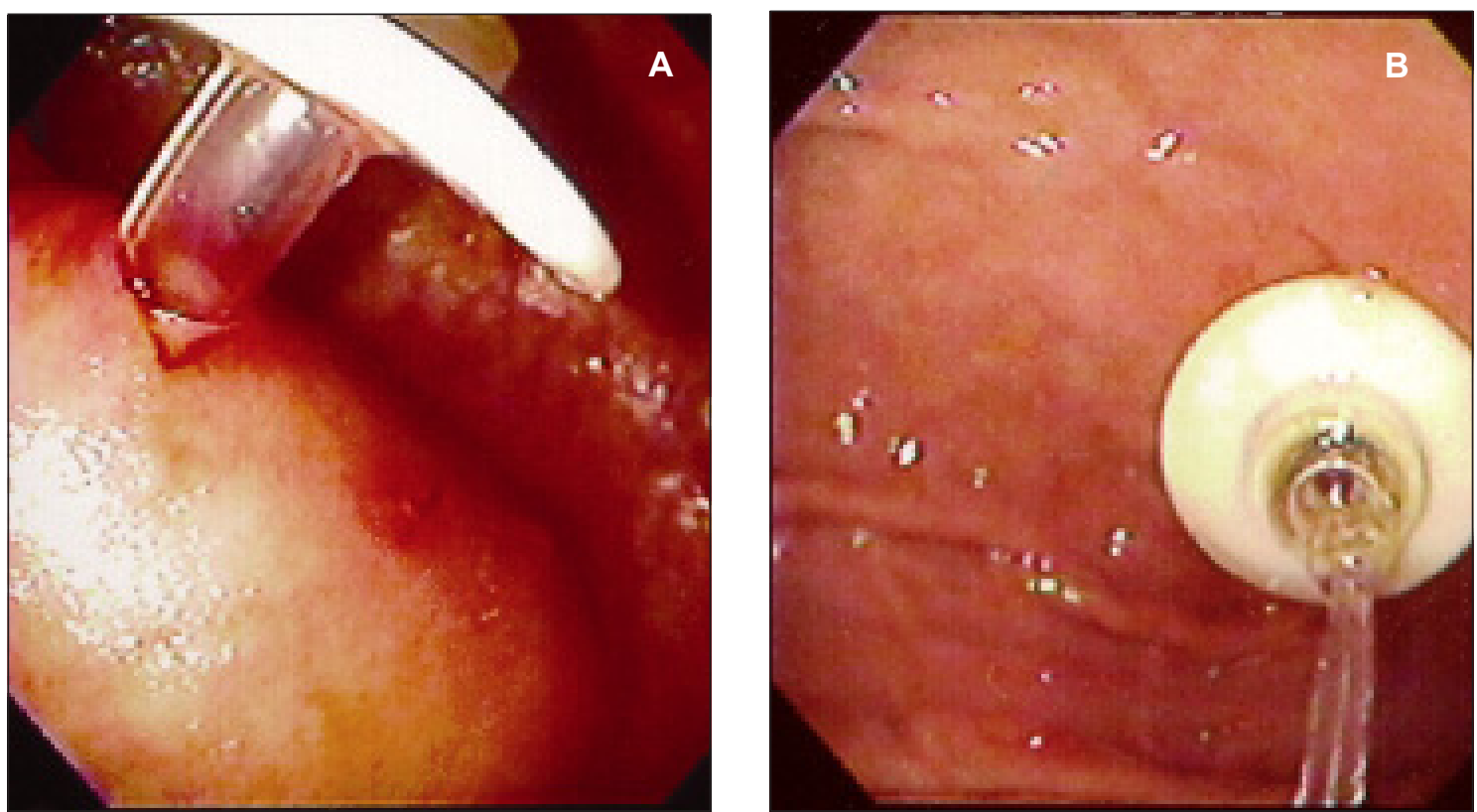

Figura 3. Aspecto endoscópico da parede do estômago. A e B - visão do disco de retenção.

da sonda encoste suavemente à parede gástrica (sob visão endoscópica - Figura 3). Finalmente, faz-se a fixação da sonda à parede abdominal externa.

Existem dois tipos principais de cateteres usados atualmente: o de poliuretano (Fresenius ${ }^{\circledR}$ ) e o de silicone (Willson-Cook ${ }^{\circledR}$ ). A comparação

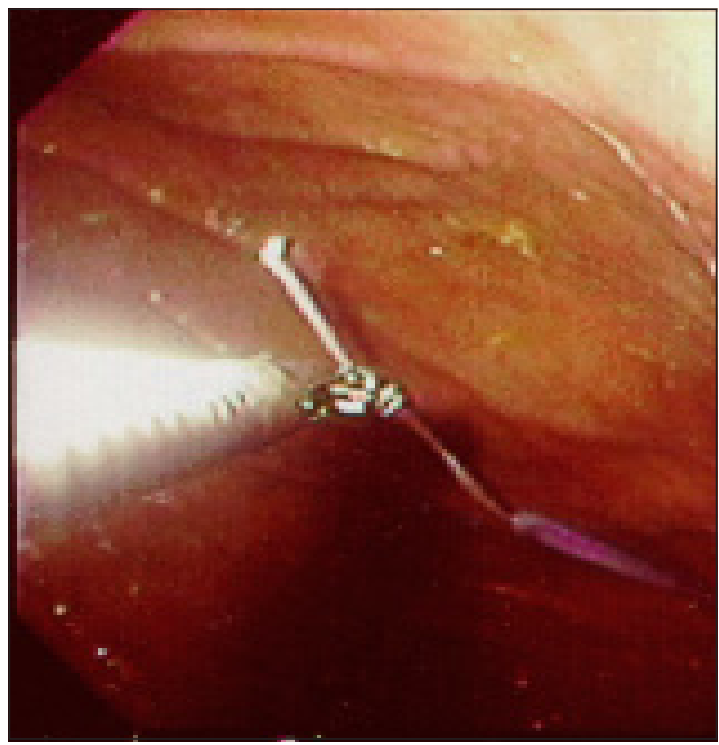

Figura 2. Fio duplo introduzido por agulha de punção e pinça de biópsia prendendo o fio. 
entre esses materiais evidenciou que o uso de sondas de poliuretano está associado ao menor número de intercorrências a curto prazo. Com relação à freqüência de complicações, de infecção local e de hemorragia, e ao tempo de funcionamento da gastrostomia, não foram observadas diferenças entre os pacientes com cateter de poliuretano e com o de silicone ${ }^{9}$.

É recomendação das sociedades francesa e americana de gastroenterologia endoscópica a antibioticoterapia profilática para a GPE, pois reduz significativamente a taxa de infecção local ${ }^{10,11}$. O regime antibiótico ideal ainda não foi estabelecido. Entretanto, Mohammed et al. ${ }^{12}$ acreditam que o uso de antibiótico profilático aumenta o risco de resistência bacteriana, principalmente para o $S$. aureus meticilina resistente. Assim preconizam o uso de antibióticos profiláticos apenas em hospitais com grande incidência de sepsis relacionada ao procedimento ${ }^{12}$.

Tradicionalmente, a utilização da GPE é iniciada 24 horas após sua realização, entretanto, Srinivasan \& Fisher ${ }^{13}$ concluíram que o início precoce da alimentação, após três horas do procedimento, é eficaz e reduz, assim, o tempo de internação hospitalar.

A GPE apresenta algumas vantagens em relação aos métodos tradicionais de suplementação que utilizam dieta enteral. É um procedimento comparado à gastrostomia cirúrgica, sem necessidade de anestesia e de laparotomia, com menor tempo de hospitalização, além do maior conforto para o paciente que o obtido com sondas nasogástricas e nasoenterais. A GPE evita processos traumáticos e erosivos da nasofaringe e esôfago, sendo esteticamente mais aceita; ao compararmos com a sonda nasoenteral, a perda da sonda e o deslocamento de sua ponta são minimizados na GPE. Essa via de acesso não exige restrição física ou uso de sedativos para manutenção da via de administração da terapia nutricional, visto que a remoção das sondas nasoenterais é muito freqüente, principalmente nas doenças neurológicas ${ }^{14}$. Além disso, a GPE proporciona custos menores em relação à técnica cirúrgica ${ }^{15}$, pois pode ser realizada tanto em centro cirúrgico como no aposento do paciente, representando economia ao redor de $40 \%$. 0 preço de cada sonda varia de trezentos e cinqüenta a quatrocentos e cinqüenta reais; contribui também para a redução dos custos do procedimento a possibilidade do paciente ter alta no mesmo dia de sua inserção. Com essa metodologia não foram observados aumentos nas taxas de complicações e nas de morbimortalidade ${ }^{16}$. É descrito que os pacientes com doença do neurônio motor e portadores de acidente vascular encefálico apresentaram diminuição da mortalidade e melhora da qualidade de vida, quando submetidos a GPE ${ }^{4}$.

As complicações relacionadas à GPE podem ser divididas em menores e maiores ${ }^{2}$ ou nas relacionadas e não relacionadas com o tubo ${ }^{17}$. As complicações ${ }^{2}$ menores ocorrem em 7\% a 10\% dos casos e estão relacionadas com: infecção local da pele, a mais comum; obstrução do tubo; remoção acidental do tubo; dor abdominal secundária, principalmente peritonite química². As complicações maiores são raras, ocorrendo em $1 \%$ a $2 \%$ dos casos $^{2}$; entre elas destacam-se: óbito; fasceíte necrosante; pneumonia aspirativa; sangramento grave; peritonite; fístula gastrocólica; perfuração; disseminação tumoral.

O índice de mortalidade é de 0,3\% a 1,0\% e está normalmente associado com a idade dos pacientes e suas co-morbidades².

As complicações relacionadas à sonda são: migração, obstrução da mesma, fístula, disseminação tumoral, íleo paralítico e peritonismo. Aquelas não relacionadas à sonda incluem infecções locais e refluxo gastroesofágico. As que ocorrem durante o procedimento são: perfuração de esôfago, hipoventilação decorrente da sedação e pneumonia aspirativa ${ }^{17}$. Cukier et al. ${ }^{18}$, em estudo em que a GPE foi praticada em cardiopatas 
com isquemia cerebral e que necessitavam de nutrição enteral, não observaram complicações durante o procedimento. A maior dificuldade foi a localização do corpo gástrico, e apenas um entre doze pacientes apresentou infecção do orifício cutâneo de inserção do equipamento ${ }^{18}$.

Grande parte dos cateteres necessitará de recolocação devido à ruptura, deterioração, oclusão e descolamento. Se ocorrer remoção acidental do tubo até catorze dias após sua colocação e o paciente não apresentar sinais ou sintomas de peritonite, sugere-se apenas observação e colocação de nova sonda em cinco a sete dias. Não se recomenda passagem de novo cateter por pertuito imaturo. Se houver sinais de peritonite, a exploração da cavidade abdominal faz-se necessária, com fechamento da gastrostomia, irrigação da cavidade peritoneal e colocação de novo acesso enteral ${ }^{17}$. O deslocamento e a remoção da sonda podem ser minimizados com a colocação do botão de GPE que, diferentemente do equipamento tradicional, não apresenta sonda externa ${ }^{19}$. Existem dois tipos principais de cateter para serem colocados após a perda da GPE: um deles com balão e duplo lúmen e o segundo sem balão e apenas um lúmen. A perda desses cateteres ocorre em $46 \%$ dos casos por deterioração do tubo, seguida pela obstrução dos mesmos quando utilizado o de lúmen único e sem balão. Com o de duplo lúmen, $56 \%$ das perdas ocorrem por ruptura do cateter seguida por deterioração e desinsuflação do balão. Segundo Heiser \& Malaty20, não há evidências de qual cateter seja mais efetivo e qual traz menores complicações.

Para reduzir complicações e aumentar a vida média da sonda, medidas devem ser adotadas: assepsia do local da punção com água e sabão e realização de curativo tópico, lavagem da sonda com $30 \mathrm{~mL}$ de água morna após administração de nutrientes, evitar utilização de líquidos ácidos, bebidas alcoólicas, e, em caso de obstrução do lúmen, não tentar desobstruir com pressão nem com o mandril, devendo a sonda ser trocada.
Os objetivos da GPE nem sempre são atingidos, como observado em pacientes demenciados, não havendo dados na literatura mostrando esses benefícios. Diminuição da mortalidade não foi evidenciada e Skelly, em artigo de revisão, descreve até evolução desfavorável nesses casos, quando submetidos ao procedimento ${ }^{4}$. A manutenção do estado nutricional com o uso da GPE também não tem sido confirmada, devido, principalmente, à inadequação calórica que ocorre por complicações mecânicas (perda do cateter), diarréia e incremento catabólico de processos inflamatórios agudos ${ }^{21}$. Entretanto, mesmo com ganho de $3,5 \mathrm{~kg}$ em quatro anos, foi observada por Loser et al. ${ }^{22}$ alta mortalidade (66\%) no primeiro ano após o procedimento. Assim, de acordo com Ciocon et al..$^{23}$, preconizamos que o objetivo do procedimento deva ser a estabilização do peso e da albumina ao invés da recuperação do estado nutricional.

Além disso, a incidência de pneumonia aspirativa também não diminuiu com a introdução da GPE ${ }^{24}$, o mesmo acontecendo com as escaras de decúbito e infecções, sendo que essas últimas encontram-se até aumentadas em pacientes que foram submetidos ao procedimento ${ }^{25}$. Dharmarajan et al. ${ }^{25}$ mostraram que a GPE pode levar os pacientes com alguma deglutição residual ao isolamento social, à depressão e, principalmente, à privação do prazer da alimentação via oral 25 .

Logo, restam dúvidas se a GPE apenas prolonga a vida ou melhora a qualidade de vida dos pacientes a ela submetidos 26,27 .

A colocação de um cateter de gastrostomia é considerada tratamento médico pela Associação Médica Americana, visto que por meio dela o paciente receberá alimentação e hidratação. Logo, como todo tratamento médico, pode ser negado pelo paciente, pois passa pela sua autonomia ${ }^{25}$. Em estudo realizado por Brett et al. ${ }^{28}$, o procedimento foi autorizado pelos familiares em 92,2\% dos casos, e em menos de 1,0\%, riscos, benefícios ou outras opções foram discutidas com os 
pacientes ${ }^{29}$. Em situações em que o paciente perde a capacidade de tomar decisões, essas acabam sendo transferidas para a família.

Em resumo, a GPE é um procedimento novo que pode trazer vantagens sobre os métodos de gastrostomia até então empregados. Sua indicação em nosso meio vem aumentando gradativamente, possibilitando discussões mais aprofundadas de suas indicações, riscos, benefícios e a participação de paciente e familiares nesse processo.

\section{REFERÊ NCIAS}

1. Gauderer MWL, Ponsky JL, Izanti RJ. Gastrostomy without laparotomy a percutaneous endoscopic technique. J Paediatr Surg. 1980; 15(6):872-5.

2. Milkes DE. Percutaneous endoscopy gastrostomy (GPE) tubes: medical, legal and ethical considerations for patients and physicians [cited 2002 Feb 20]. Available from: www.ciberouds. com/conferences/gastroenterology/current/ conference, 2000

3. Rombeau JL. Enteral nutrition. In: Goldman L, Bennett JC, editors. Cecil textbook of medicine. 21th ed. Philadelphia: WB Saunders Co; 2000. p.1163-6.

4. Skelly RH. Are we using percutaneous endoscopic gastrostomy appropriately in the elderly? Curr Opin Clin Nutr Metab Care. 2002; 5(1):35-42.

5. Nicholson FB, Korman MG, Richardson MA. Percutaneous endoscopic gastrostomy: a review of indications, complications and outcomes. J Gastroenterol Hepatol. 2000;15(1):21-5.

6. Fein PA. Safety of percutaneous endoscopic gastrostomy tubes in peritoneal dialysis patients. Semin Dial. 2002; 15(3):213-4.

7. Taylor AL, Carroll TA, Jakubowski J, O’Reilly G. Percutaneous endoscopic gastrostomy in patients with ventriculoperitoneal shunts. Br J Surg. 2001; 88(5):724-7.

8. Brimacombe J, Newell S, Bergin A, Mccarthy J, Barry J. The laryngeal mask for percutaneous endoscopic gastrostomy. Anesth Analg. 2002;91(3):635-6.

9. Hazel SJVD, Mulder CJJ, Hartog G, Thies JE, Westhof W. A randomized trial of polyurethane and silicone percutaneous endoscopic gastrostomy catheters. Aliment Pharmacol Ther. 2000; 14(10):1273-7.
10. Preclik G, Grune S, Leser J, Lebherz W, Machka K, Holstege A. Prospective, randomised, double blind trial of prophylaxis with single dose of co- amoxiclav before percutaneous endoscopic gastrostomy. BMJ. 1999; 319(7214):881-4.

11. Jain NK, Larson DE, Schroeder KW. Antibiotic prophylaxis for percutaneous endoscopic gastrostomy. A prospective, randomized, double-blind clinical trial. Ann Intern Med. 1987; 107(6): 824-8.

12. Mohammed I, Jones BJ. Antibiotic prophylaxis after percutaneous endoscopic gastrostomy insertion: widespread routine use of prophylactic antibiotics might predispose to increased risk of resistant organisms. Br Med J. 2000; 320(7238): 870-1.

13. Srinivasan R, Fisher R. Early initiation of post-GPE feeding: do published recommendations affect clinical practice? Dig Dis Sci. 2000; 45(10): 2065-8.

14. Verhoef MJ, Van Rosendaal M. Patient outcomes related to percutaneous endoscopic gastrostomy placement. J Clin Gastroenterol. 2001; 32(1): 49-53.

15. Harbrecht BG, Moraca RJ, Saul M, Courcoulas AP. Percutaneous endoscopic gastrostomy reduces total hospital costs in head-injured patients. Am J Surg. 1998; 176(4):311-4.

16. Mandal A, Steel A, Davidson AR, Ashby C. Day-case percutaneous endoscopic gastrostomy: a viable proposition? Postgrad Med J. 2000; 76(893):157-9.

17. Siddique I, Krishnamurthy M, Choubey S, Gudavalli P, Bharathan T, Pachter B. Colocutaneous fistula: a rare and silent complication of percutaneous endoscopic gastrostomy. Dig Dis Sci. 1996; 41(2):301-4.

18. Cukier C, Magnoni CD, Poletti P, Tacla M. Gastrostomia endoscópica em pacientes com cardiopatia complicada. Arq Gastroenterol. 2000; 37(4):208-12.

19. Muramatsu H, Koike K, Teramoto A. Benefits of percutaneous endoscopic button gastrostomy in neurological rehabilitation therapy. Int J Rehabil Res. 2002; 25(2):157-61.

20. Heiser M, Malaty $H$. Ballon-type versus non-balloon-type replacement percutaneous endoscopic gastrostomy: which is better? Gastroenterol Nurs. 2001; 24(2):58-63.

21. Cefalu CA. Appropriate dysphagia evaluation and management of the nursing home pacient with dementia. Ann Long Term Care. 1999; 7(12): 447-51. 
22. Loser C, Wolters S, Folsch UR. Enteral long-term nutrition via percutaneous endoscopic gastrostomy in 210 patients. Dig Dis Sci. 1998; 43(11):2549-57.

23. Ciocon JO, Silverstone FA, Graver M, Foley CJ. Tube feeding in elderly patients: indications, benefits, and complications. Arch Intern Med. 1988; 148(2):429-33.

24. Kim Y. To feed or not to feed: tube feeding in patients with advanced dementia. Nutr Rev. 2001; 59(3):86-8.

25. Dharmarajan TS, Unnikrishnan D, Pitchumoni CS. Percutaneous endoscopic gastrostomy and outcome in dementia. Am J Gastroenterol. 2001; 96(9):2556-63.
26. Finucane TE, Christmas C, Travis K. Tube feeding in patients with advanced dementia: A review of the evidence. JAMA. 1999; 282(14):1365-70.

27. Gillick MR. Rethinking the role of tube feeding in patients with advanced dementia. N Engl J Med. 2000; 342(3):206-10.

28. Brett AS, Rosenberg JC. The adequacy of informed consent for placement of gastrostomy tubes. Arch Intern Med. 2001; 161(5):745-8.

29. Bourne D. Informed Consent and the placement of percutaneous endoscopic gastrostomy tubes. Arch Intern Med. 2001; 161(20):2506-7.

Recebido para publciação em 9 de abril de 2003 e aceito em 6 de abril de 2004. 